\title{
Zur Theorie der Turgordehnung und über den funktionellen Zusammen- hang zwischen den einzelnen osmotischen Zustandsgrößen
}

\author{
Von
}

Hiroshi Tamiya

(Mitteilung aus dem botanischen Institut der kaiserl. Universität zu Tokyo, und dem Tokugawa Biologischen Institut)

Ëngegangen ant 26. Seftemlicr 19.37

Bei der statischen Betrachtung der osmotischen Zustandsgrößen kommt bekanntlich die grundlegende Formel zur Geltung:

Osmotischer Wert des Zellinhaltes = Saugkraft der Zelle+Turgordruck.

Diese Formel gibt ja einwandfrei die summarische Beziehung der osmotischen Zustandsgrößen wieder, kann aber nichts darüber aussagen, ob und in welchem funktionellen Verhältnis die einzelnen in Betracht kommenden Werte zueinander stehen. Diese Frage, die offenbar für die Theorie der osmotischen Erscheinungen von großer Bedeutung ist, wurde schon von A. URsPRUNG (1926) näher bearbeitet, der aber endlich zu dem Schluß kam, daß dabei ,gesetzmäßige Beziehung von allgemeiner Gültigkeit nicht zu erkennen sind.“ Ferner sagt er: „Für die Technik der osmotischen Untersuchungen ergibt sich die Regel, daß man den Zahlenwert einer bestimmten osmotischen Größe nicht aus dem Verhalten einer anderen Größe ableiten kann." Im folgenden soll aber gezeigt werden, dap dieser negative Beschluß von URsPRUNG, zumindest bezüglich der einfachst gebauten Zellen, wohl nicht ganz aufrechtzuhalten ist, und zwar, daß in gewissen Fällen wirklich eine regelmäßige funktionelle Beziehung zwischen den einzelnen osmotischen Zustandsgrößen bestehen kann.

Neulich erschien eine interessante Arbeit von T. SaKamura (1937), in welcher er eine sehr aufschlußreiche Methode zur schematischen Darstellung der gegenseitigen Beziehungen der osmotischen Zustandsgrößen vorbrachte. Da mein Gedankengang am besten durch dieses Diagramm veranschaulicht werden kann, sei hier zunächst eine kurze Bemerkung darüber gemacht. Man trägt in einem Koordinatensystem den osmotischen Wert der Zelle auf die Ordinate, und denjenigen der mit dieser Zelle im Gleichgewicht stehenden Aupenlösung auf die Abszisse auf (Fig. 1). Es sei nun:

$C_{e n}$ : osmotischer Wert des Zellinhaltes,

$C_{\rho y}$ : osmotischer Wert der Außenlösung,

$C_{i}$ : osmotischer Wert des Zellinhaltes bei Grenzplasmolyse = osmotischer Wert der Außenlösung bei Grenzplasmolyse. 
Der osmotische Wert der Außenlösung sei an dem Punkt $B$ null, und an $C$ gleich dem plasmolytischen Grenzwert $C_{i}$, wobei also auch $C_{e n}=C_{i}$ ist. Auf der Linie $B D$, die die Diagonale des Quadrates $A B C D$ darstellt, ist $C_{e n}$ stets gleich $C_{e x}$, was nur bei einer hypothetischen Zelle, deren Wand ohne jeglichen Widerstand ideal dehnbar ist, der Fall sein kann. Dagegen ist auf der Linie $A D$ der Wert $C_{c n}$ stets unabhängig von $C_{e x}$ gleich $C_{i}$, also ein Umstand, der bei den Zellen mit vollkommen fester Wand zur Beobachtung kommen soll. In Wirklichkeit ist aber die Zellwand weder ideal dehnbar noch vollkommen rigid, sodaß die $C_{e n}$ $C_{e x}$-Kurve immer zwischen $B D$ und

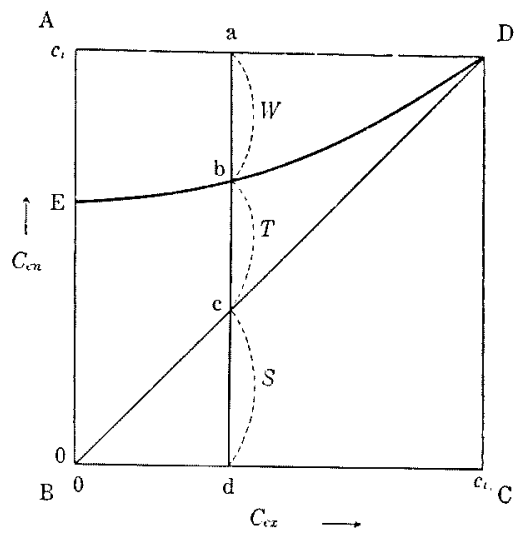

Fig. 1 $A D$, etwa so wie die Kurve $D E$, liegen muß. Ob und wie diese Kurve gestaltet ist, hängt von der Natur der Zellwand ab. Steht nun eine Zelle mit einer Außenlösung im Gleichgewicht, welche letztere einen dem Punkt $d$ entsprechenden osmotischen Wert aufweist, so bedeutet, wie schon von SaKamura näher erörtert:

Linie $b d$ : osmotischen Wert des Zellinhaltes: $C_{c n}$,

Linie $b c$ : Turgordruck: $T$,

Linie $c d$ : Saugkraft der Zelle: $S$.

$S$ und $C_{e x}$ müssen im Gleichgewicht gleich ausfallen, weil die beiden Größen wasseranziehende Potentiale der Zelle bzw. der Außenlösung bedeuten, und deshalb sie bei Einstellung des Gleichgewichts miteinander eben Wage halten müssen.1

Es lautet also nach der Grundformel der osmotischen Zustandsgrößen :

$$
C_{e n}=T+C_{e x}=T+S
$$

Was nun die Strecke $a b$ anbelangt, so entspricht sie dem osmotischen Wert der Zelle, der bei Überführung der Zelle von grenzplasmolytischem Zustand zu betreffendem Zustand wegen Wasseraufnahme verloren geht, oder anders ausgedrückt, stellt sie denjenigen osmotischen Wert dar, den die Zelle bei dieser Zustandsänderung zur Wassersaugung aufwendet. Diesen Wert, der für unsere weitere Auseinandersetzung von Bedeutung ist, wollen wir "Wasserwert"

1) Eben hierin besteht das Prinzip der sogen. II. Methode (Gleichgewichtsmethode) der Saugkraftbestimmung nach A. UrSPrung und G. Blum. 
nennen und mit $W$ bezeichnen. Wie aus dem Gleichgewichtsdiagramm ersichtlich besteht folgende Beziehung:

$$
\begin{aligned}
W & =C_{i}-C_{e n} \\
& =C_{i}-(T+S)
\end{aligned}
$$

Bezeichnen wir ferner mit

$C_{w}$ : osmotischen Wert des Zellinhaltes im wassergesättigten Zustand $\left(C_{c,}=0\right)$,

$V_{\psi}$ : Volumen der Zelle im wassergesättigten Zustand,

$V_{i}$ : Volumen der Zelle bei Grenzplasmolyse,

$V: \quad$ Volumen der Zelle, deren osmotischer Wert $C_{e n}$ ist,

so darf man folgende Formel (Gesetz von BoyLe-MariotTe) hinstellen, vorausgesetzt, daß die Zelle eine große Zentralvakuole und eine genügend dünne protoplasmatische Schicht besitzt.1)

$$
C_{w} V_{w}=C_{e n} V=C_{i} V_{i}
$$

Die Formel (II) läßt sich danach wie folgt umschreiben,

$$
W=C_{i}\left(1-\frac{V_{i}}{V}\right)
$$

Bei einer Zelle stellt $V_{i}$, soweit kein Wachstum stattindet, stets eine bestimmte Größe dar; ebenso ist $C_{i}$ insofern konstant, als die Menge der osmotisch aktiven Substanzen im Zellsaft unverändert bleibt.

Unser Interesse gilt nun, den Verlauf der $C_{e n}-C_{c .}$-Kurve, d.i. die Veränderung des $W$-Wertes in Funktion von $C_{c x}$, zu analysieren. Da $W$ nach der Formel (IV) mit $V$ verbunden ist, so kommt es auch darauf an, den Verlauf der Volumenänderung analytisch zu verfolgen. Schon früher haben A. URsPrung und G. BLum (1916) angenommen, da13 zwischen der Zunahme des Turgordrucks und der des Zellvolumens eine einfache Proportionalität:

$$
\frac{d T}{d V}=\text { Const. }
$$

besteht. ${ }^{2)}$ Diese Annahme stützte sich auf dem Befund bei einem Modellversuch mit einem Kautschukschlauch, dessen Elastizitätseigenschaft nach der Ansicht der genannten Autoren als identisch mit derjenigen der Zellmembran angesehen werden dürfte. Durch

1) Vergl. K. HöFler (1917a).

2) Vergleich auch A. Ursprung (1932) u. K. Höfler (1920). 
Integration der obigen Formel ergibt sich (weil bei $C_{c x}=C_{i} \quad T=0$ ist) :

$$
T=k\left(V-V_{i}\right)
$$

worin $k$ die Proportionalitätskonstante darstellt. Da im Wasser der Turgordruck der Zelle gleich $C_{w}$ ist (weil dabei $S=0$ ist), so muß nach (2)

$$
C_{w}=k\left(V_{w}-V_{i}\right)
$$

sein, woraus weiter folgt:

$$
\begin{aligned}
& k=\frac{C_{w}}{V_{w}-V_{i}}, \\
& T=\frac{C_{w}\left(V-V_{i}\right)}{\left(V_{w}-V_{i}\right)}
\end{aligned}
$$

Aus (3), (I) und (III) ergibt sich:

$$
\begin{aligned}
\frac{C_{i} V_{i}}{V}-C_{e x} & =\frac{C_{i} V_{i}\left(V-V_{i}\right)}{V_{w}\left(V_{w}-V_{i}\right)} \\
\text { oder } \quad \frac{1}{\phi}-\frac{C_{e x}}{C_{i}} & =\frac{(\phi-1)}{\phi_{w}\left(\phi_{w}-1\right)}
\end{aligned}
$$

wobei $\phi$ für $\frac{V}{V_{i}}$, und $\phi_{w}$ für $\frac{V_{w}}{V_{i}}$ steht. $\phi_{w}$ ist eine je nach den Arten der Zelle bestimmte, durch die Dehnbarkeit der Zellwand bedingte Konstante, die experimentell ermittelbar ist. Setzt man

$$
\phi_{w}\left(\phi_{w}-1\right)=K,
$$

wobei stets $K>1$ ist, so erhalten wir aus (4) die Beziehung:

$$
\phi^{2}-\phi+K \frac{C_{e x}}{C_{i}} \phi-K=0
$$

Daraus folgt:

$$
\begin{gathered}
\frac{d \phi}{d\left(C_{i}-C_{e x}\right)}=\frac{K}{\frac{C_{i} K}{\phi^{2}}+C_{i}} \\
\phi=\frac{1}{2 C_{i}}\left[C_{i}-K C_{e x}+\sqrt{K^{2} C_{e x}^{2}-2 K C_{e x} C_{i}+C_{i}^{2}+4 K C_{i}^{2}}\right]
\end{gathered}
$$

Dies sind die Beziehungen, die zwischen dem Grad der Turgordehnung $\phi$ und dem osmotischen Wert der Aupenlösung bestehen sollten, wenn die mit der Formel (1) ausgedrückte Annahme URSPRUNGs richtig wäre. Trägt man $\phi$ auf die Ordinate und $C_{i}-C_{e x}$ auf die Abszisse auf, so erhält man nach obiger Formel eine Kurve, die, wie in Fig. 2 gezeigt, stets nach oben konkav aufsteigt.

Nun liegen in der Literatur eine Anzahl der Arbeiten vor, in 
denen der Grad der Turgordehnung unter dem Einfluß variierender Konzentration der Außenlösung quantitativ verfolgt wurde. ${ }^{1)}$ Inso-

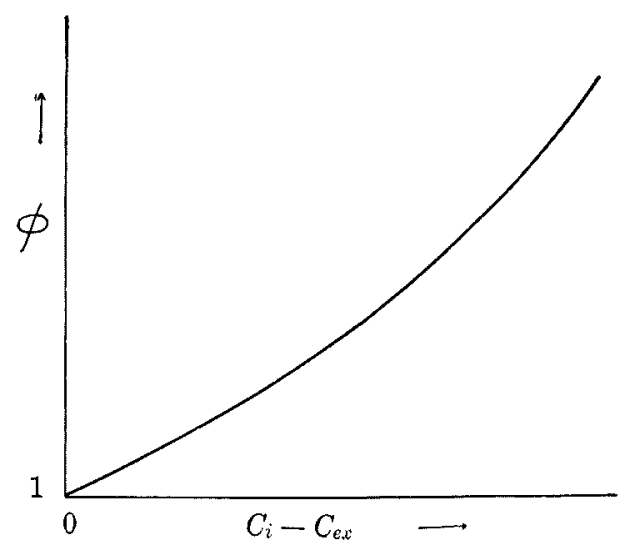

Fig. 2 fern als es sich um pflanzliche Zellen handelt, stimmen die Befunde von ver. schiedenen Autoren ganz ausnahmslos darin überein, daß die Zelle, die bei Grenzplasmolyse völlig entspannt ist, mit der Abnahme von $C_{e x}$ (also mit der Zunahme von $C_{i}-C_{e x}$ ) zunächst ganz auffallende aber dann immer undeutlichere Dehnung aufweist. So ist die der Einheit der Abnahme von $C_{e x}$ bezogene Dehnung stets am größten in der Nähe der Grenzplasmolyse, sinkt aber mit der Abnahme von $C_{e x}$ allmählich, und zwar asymptotisch gegen Null ab, kurz ist die $\phi_{-}\left(C_{i}-C_{c x}\right)$-Kurve stets mehr oder weniger gegen die Abszissenachse gekrümmt, wie es in Fig. 3 dargestellt ist.")

Angesichts dieser Tatsache wird es einleuchtend, daß die Annahme von URSPRUNG und BLUM nicht stichhaltig ist. Schon hat OPPENHEIMER (1930) darauf aufmerksam gemacht, daß man die Dehnbarkeit

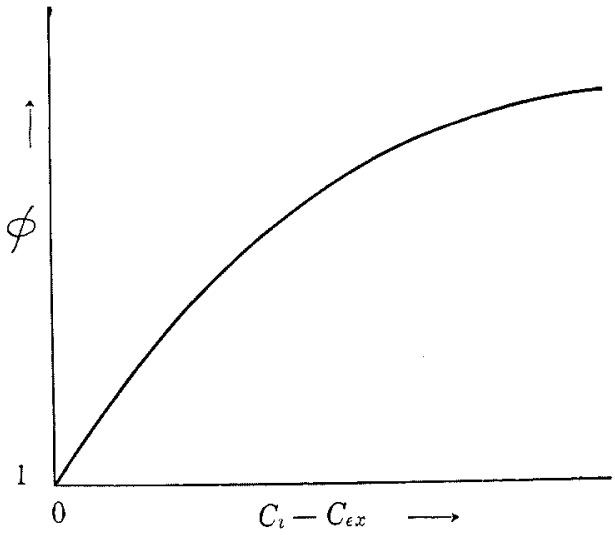

Fig. 3

1) W. W. LEPESCHKIN (1907): Spirogyra.

H. R. OPPENHEIMER (1930): Wurzelrinde von Allium cepa, Markzelle des Infloreszenzstiels von Taraxacum officinale, Epidermis von Tradescantia zebrina.

\section{STow (1936): Nitella.}

F. v. HoFe (1933): Lepiota u.a.

C. Hofrmann (1935): Bangia, Chaetomorpha, Elachista, Callitham-

2) Bei den Untersuchungen, in denen nicht die Dehnung der einzelnen Zellen, sondern etwa lineale Turgordehnung des Gewebestreifens gemessen wurde [z.B. F. v. Hore (1933), E. G. Pringsheim (1931), H. Gamma (1932)], sind $\mathrm{ab}$ und $\mathrm{zu}$ recht unregelmäßige, manchmal sogar nach oben gekrümmte Kurven beobachtet worden. Die Dehnung der Gewebestreifen birgt aber, wie schon von OPPENHEIMER u.a. bemerkt, eine Anzahl der Kompliziertheiten in sich, soda hier von allen solchen Fällen Abstand genommen werden soll. 
der Zellmembran nicht, wie es bei URSPRUNG und BLUM geschah, mit derjenigen des Kautschukschlauchs identifizieren darf. Tatsächlich ist die Zellwand viel weniger dehnbar wie der Kautschuk, indem das Hoocksche Proportionalitätsgesetz, welchem weitgehend elastische Körper wie Kautschuk gehorchen, nur begrenzt in ganz kleiner Spanne der Spannung Gültigkeit hat. ${ }^{1)}$ A. N. J. HEYN (1933), der die Dehnbarkeit der Avena-Koleoptile durch Belastungsversuch untersuchte, stellte fest, daß die Dehnung zuerst mit weniger Belastung sehr deutlich erfolgt, aber allmählich mit der Zunahme der Spannung sich ein immer zunehmender Widerstand gegen weitere Dehnung bemerkbar macht. Die Gewicht-Dehnung-Kurve verläuft dabei im Grunde analog der $\phi_{-}\left(C_{i}-C_{e x}\right)$-Kurve, die überall bei Turgordehnung der Zellen zur Beobachtung kommt.

Wie sollte nun dieses allgemeine Charakteristikum der Membrandehnung im Gestalt der $C_{e n}-C_{e x}$-Kurve in unserem Gleichgewichtsdiagramm zum Ausdruck kommen? Zunächst ist hervorzu-

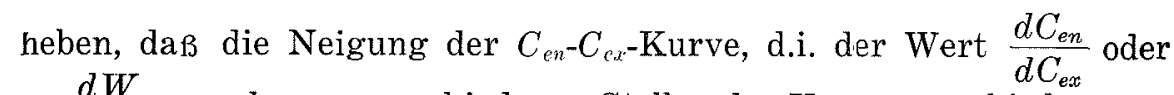
$\frac{d W}{d\left(C_{i}-C_{e x}\right)}$, der an verschiedenen Stellen der Kurve verschieden ausfällt, ein Maß der Dehnbarkeit der Zellwand am betreffenden Zustand darstellt. Ist bei einem Zustand die Zellwand vollständig fest, so ist $\frac{d W}{d\left(C_{i}-C_{e x}\right)}$ null, während bei vollständig turgeszenzlosem Zustand $\frac{d W}{d\left(C_{i}-C_{e x}\right)}=1$ sein kann. Erfahrungsgemäß ist die Dehnbarkeit der Zellmembran in der Nähe der Grenzplasmolyse am größten $\left(\frac{d W}{d\left(C_{i}-C_{e x}\right)} \rightarrow 1\right)$, während sie mit der Zunahme des Turgeszenzgrades, d.h. mit dem Sinken der Außenkonzentration, asymptotisch gegen Null $\left(\frac{d W}{d\left(C_{i}-C_{e x}\right)} \rightarrow 0\right)$ absinkt. Die Veränderung von $W$ in Funktion von $C_{i}-$ $C_{e x x}$ läßt sich also etwa so darstellen wie die vollausgezogene Kurve in Fig. 4, vorausgesetzt:

1) daß, im Laufe der Turgordehnung keine plötzliche, qualitative Veränderung der Mem-

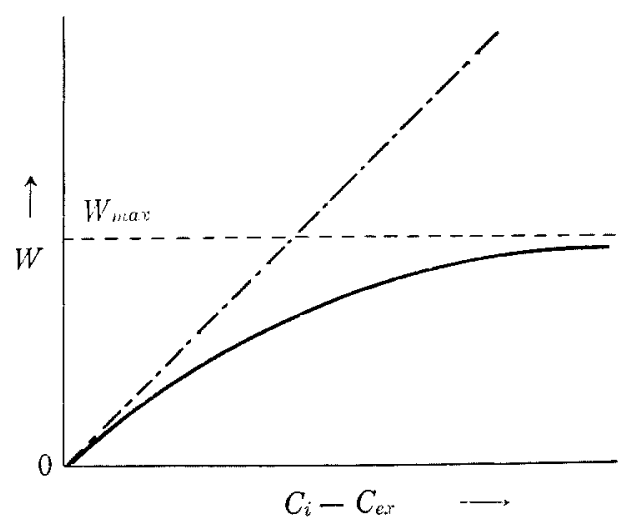

Fig. 4

1) Vergl. z.B. L. Buch (1935), A. Frey-Wyssling (1935). 
braneigenschaften, wie Zerreißen, übermäßige plastische Dehnung oder Plasmolyse stattfindet, und

2) daß keine Kompliziertheiten wegen der polaren Differenzierung der Membraneigenschaften (etwa die Verschiedenheit der elastischen Eigenschaft von Quer- und Längswand) in Erscheinung treten.

Bezeichnet man die maximale Grenze des Wasserwertes, zu welcher $W$ asymptotisch nähert, mit $W_{m a x .}$, so ist der Verlauf der Kurve wohl am einfachsten durch folgende Formel auszudrücken.

$$
\frac{d W}{d\left(C_{i}-C_{e x}\right)}=f\left(W_{\max }-\mathrm{W}\right)
$$

worin $f$ eine Konstante darstellt. Schreibt man ferner

so ist

$$
f W_{\max }=\alpha
$$

$$
\frac{d W}{d\left(C_{i}-C_{e x}\right)}=\alpha-f W
$$

$\alpha$ ist der Wert von $\frac{d W}{d\left(C_{i}-C_{e x}\right)}$ bei der Grenzplasmolyse, weil dabei $W=0$ ist, und gleich der Tangente des Winkels, den die $W$-Kurve am Koordinatenanfangspunkt mit der Abszissenachse einschließst. Bei parenchymatischen Zellen darf man annehmen, daß die Zellwand beim Einsetzen der Grenzplasmolyse praktisch ganz widerstandslos wird. So ist in solchen Fällen $a=1$, und die Formel $\left(V^{\prime}\right)$ geht über in:

$$
\frac{d W}{d\left(C_{i}-C_{e x}\right)}=1-f W
$$

Die Integration führt für die Anfangsbedingung $C_{i}-C_{e x}=0$, $W=0$ zu der Gleichung:

$$
W=\frac{1}{f}\left[1-e^{-f\left(C_{i}-C_{e x}\right)}\right]
$$

Ist $f=0$, so gilt nach (VII) für alle Werte von $C_{e x}$

$$
\frac{d W}{d\left(C_{i}-C_{\theta x}\right)}=1
$$

d.i. die Zellwand ist im ganzen Bereich der Außenkonzentration ideal dehnbar. Ist dagegen $f=\infty$, so ist nach (VIII) für alle Werte von $C_{e x x}$ stets $W=0$, was also bei vollkommen undehnbaren Zellwänden der Fall ist. Die Konstante $f$ indiziert mithin den Grad der Festigkeit der Zellmembran, und stellt eine für einzelne Zelle charakteris- 
tische Größe dar. Die Dimension dieses Festigkeitskoeffizienten ist der reziproke des osmotischen Wertes, welcher letztere entweder in Mol. des Osmotikums oder in Atm. auszudrücken ist.

Aus (II) und (VIII) folgt:

$$
C_{e n}=C_{i}-W=C_{i}-\frac{1}{f}\left[1-e^{-f\left(C_{i}-C_{e x}\right)}\right]
$$

Andererseits ist nach (III)

$$
C_{e n}=\frac{V_{i}}{V} C_{i}=\frac{C_{i}}{\phi}
$$

Also ist

$$
\frac{V_{i}}{V}=\frac{1}{\phi}=1-\frac{1}{f C_{i}}\left[1-e^{-f\left(C_{i}-C_{e x}\right)}\right],
$$

oder

$$
\phi=\frac{f C_{i}}{f C_{i}-1+e^{-f\left(C_{i}-C_{e x}\right)}}
$$

Diese Gleichung drückt die Abhängigkeit des Dehnungsgrades von der Konzentration der Außenlösung aus, und steht der nach der Annahme URSPRUNGs abgeleiteten Formel (5) gegenüber. Ob unsere Grundgleichung (VII) der Wirklichkeit entspricht oder nicht, kann jetzt an Hand dieser Gleichung geprüft werden. Es drängt aber die Frage auf, ob und wie man den Festigkeitskoeffizienten $f$ bei einer gegebenen Zelle bestimmen kann. Nach (IX) und (XI) lassen sich für wassergesättigte Zellen $\left(C_{c, r}=0\right)$ folgende Formeln aufstellen.

$$
\begin{aligned}
& C_{w}=C_{i}-\frac{1}{f}\left[1-e^{-f C_{i}}\right] \\
& \phi_{w}=\frac{f C_{i}}{f C_{i}-1+e^{-f C_{i}}}
\end{aligned}
$$

Setzt man für $f C_{i}$ in der rechten Seite der Gleichung (XI') verschiedene Ziffern ein, so lassen sich die entsprechenden Werte von $\phi_{w}$ ausrechnen. Das so ermittelte exponentielle Verhältnis zwischen $f C_{i}$ und $\phi_{w}$ ist in Fig. 5 graphisch wiedergegeben. Vermittelst dieser Kurve ist es möglich, für einen beliebigen Wert von $\phi_{w}$ die entsprechende Ziffer von $f C_{i}$ auszufinden, dann ist auch $f$ ermittelbar, wenn der osmotische Grenzwert $C_{i}$ bekannt ist. Die zur Bestimmung von $f$ nötige Voraussetzung ist also die, daß uns das Verhältnis $\frac{V_{w}}{V_{i}}$ und der Wert $C_{i}$ zugleich gegeben sind.

Mit dieser Vorwegnahme wollen wir nun unsere theoretische Ausführungen an einigen experimentellen Daten auf ihre Gültigkeit 
prüfen. Leider sind hierzu die Befunde von meisten Autoren untauglich, weil dabei entweder die gleichzeitige Angabe von $C_{i^{-}}, V_{w^{-}}$

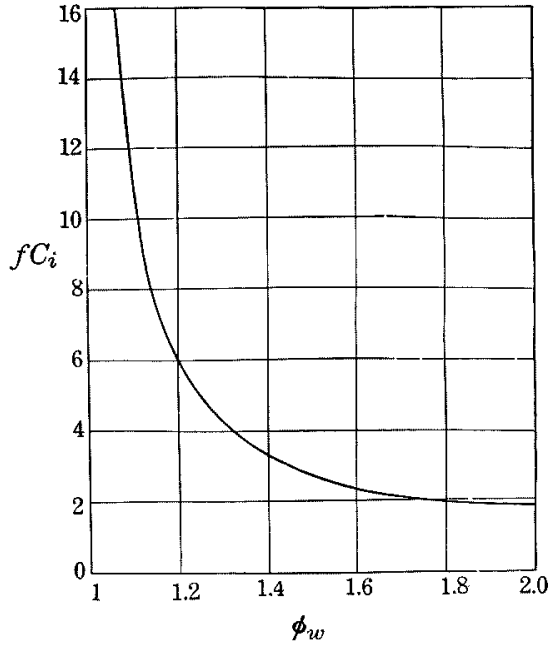

Fig. 5 und $V_{i}$-Wert fehlt, oder aber die Bestimmung des Turgeszenzgrades nur durch Vergleich der Zellänge (bei zylindrischen Zellen) oder des Zellumfangs, wodurch wir gewöhnlich keinen Aufschluß über die wirklichen Verhältnisse der Volumenänderung gewinnen können, ausgeführt worden ist. Andererseits sind auch die Angaben über die grenzplasmolytischen Werte, $C_{i}$ und $V_{i}$, oft ungenau, denn das „Einsetzen“ der Plasmolyse kann in meisten Fällen nur bei recht weit fortschreitender Plasmolyse erkannt werden. ${ }^{1)}$

Unlängst erschien eine sehr wichtige Arbeit von I. STow (1936) über die Turgordehnung von Nitella-Zellen. Dank der erheblichen Größe der Einzelzelle von dieser Alge konnte der genannte Autor zur Volumenmessung eine gravimetrische Methode anwenden, die in ihrer Exaktheit und Objektivität die anderen üblichen Methoden weit übertrifft. Ein anderer bemerkenswerter Erfolg von dieser Arbeit besteht darin, daß durch Messung der Elastizität der Zellwand in variierender Konzentration der Außenlösung der Zustand der beginnenden Isotonie von Zellsaft und Außenlösung, also der Zustand der wahren Grenzplasmolyse, sehr genau erfaßt werden konnte. Näheres über die Methodik und die Ergebnisse sei auf die Originalarbeit verwiesen, und hier, in der Tabelle 1, gebe ich davon nur die Ziffern wieder, die für unsere Darlegungen von Belang sind.2)

Tabelle 1. Turgordehung von Nitella-Zelle (I. STow)

\begin{tabular}{c|c|c}
\hline $\begin{array}{c}C_{\text {ex }} \\
\text { (Mol. } \\
\text { Rohrzucker) }\end{array}$ & $\begin{array}{c}\phi \\
\text { (gef.) }\end{array}$ & $\begin{array}{c}\phi \\
\text { (ber.) }\end{array}$ \\
\hline 0.00 & $\phi_{w}=1.40$ & 1.40 \\
0.02 & - & \\
0.04 & - & \\
0.06 & 1.37 & 1.38 \\
0.08 & 1.37 & 1.37 \\
0.10 & 1.35 & 1.35 \\
0.12 & 1.33 & 1.34 \\
0.14 & 1.31 & 1.32 \\
0.16 & 1.29 & 1.29 \\
0.18 & 1.27 & 1.27 \\
0.20 & 1.25 & 1.23 \\
0.22 & 1.20 & 1.19 \\
0.24 & 1.14 & 1.14 \\
0.26 & 1.07 & 1.08 \\
0.28 & 1.02 & 1.02 \\
$C_{i}=0.285$ & 1.00 & 1.00 \\
& &
\end{tabular}

1) Vergl. C. HoffmanN (1932).

2) Die den plasmolysierten Zustand betreffenden Ziffern sind hier nicht aufgenommen, weil wir jetzt nur die Erscheinungen bei nicht plasmolysiertem Zustand ins Auge fassen. 
Aus der Tabelle entnimmt man, daß $\phi_{w}=1.40$ ist. Durch die graphische Darstellung in Fig. 5 läßt sich feststellen, daß diese Ziffer dem $f C_{i}$-Wert von 3.40 entspricht. $\mathrm{Da} C_{i}=0.285 \mathrm{Mol}$. ist, so berechnet sich $f$ zu 11.9 (1/Mol.). Durch Einsetzen dieser Werte in der Formel (XI) habe ich für verschiedene Werte von $C_{e x}$ den Quotienten $\phi$ ausberechnet, und dies in der dritten Kolonne der Tabelle angegeben. In Fig. 6 sind die von STow gefundenen Werte

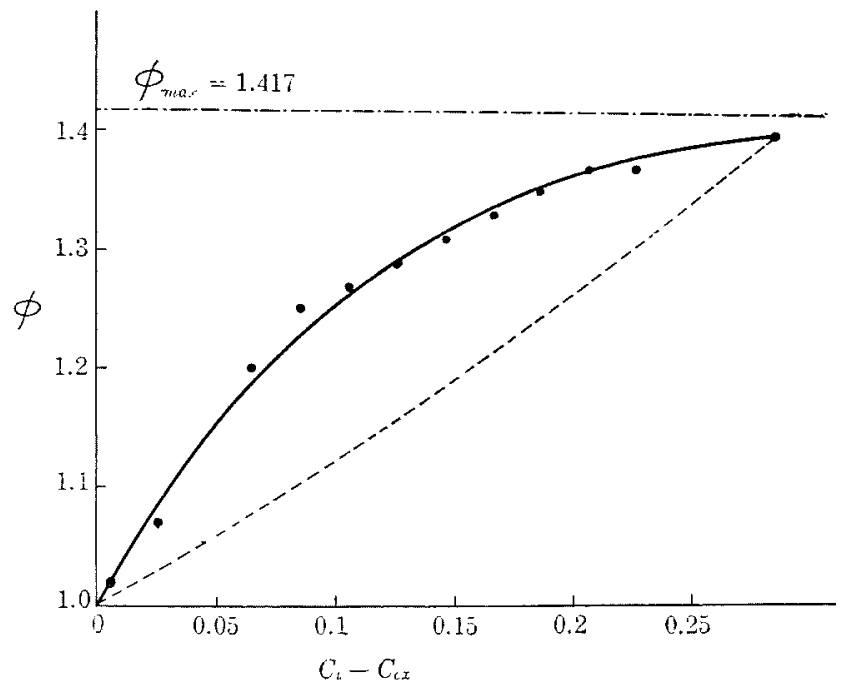

Fig. 6

mit Punkten und die von uns ausberechneten mit ausgezogener Kurve dargestellt. Die punktierte Kurve stellt die Werte dar, die sich nach der Annahme URsprungs durch die Formel (5) berechnen lassen. Es springt sofort in die Augen, daß die nach unserer Theorie erhaltene Kurve vortrefflich mit den gefundenen Ziffern übereinkommt, während sich aus der Annahme URSPRUNGS eine ganz deutlich abweichende Kurve ergibt.

Als ein anderes,
leider aber wegen mancher Gründe weniger gutes Beispiel dient die Angabe von H. R. OPPENHEIMER (1930) über die Turgordehnung der Mark-

Tabelle 2. Turgordehnung der Markzelle von Taraxacum (H. R. OPPENHEIMER)

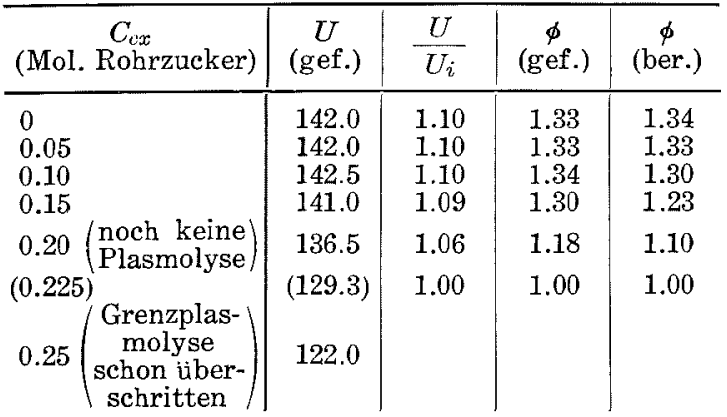

zelle von Taraxacum officinale. (Vergl. Tabelle 2). Da dieser Forscher nicht die Veränderung des Zellvolumens, sondern diejenige 
des Zellumfangs als Maßstab der Turgordehnung bestimmt hat, so bedarf hier sein Ergebnis einer gewissen Korrigierung. Obendrein wurden dabei keine genauen Werte für grenzplasmolytischen Zustand angegeben, und zwar weiß man nur, daß die Grenzplasmolyse bei $C_{e x}=0.20 \mathrm{Mol}$. (Rohrzucker) noch nicht erreicht, aber bei $C_{e: x}=$ $0.25 \mathrm{Mol}$. schon überschritten war. Es liegt nahe, als $C_{i}$ und Zellumfang $(U)$ bei der wahren Grenzplasmolyse wohl die Durchschnittswerte von den Ziffern bei $C_{e x}=0.20$ und $C_{e, x}=0.25$ anzunehmen. So ist

$$
C_{i}=0.225 \mathrm{Mol} \text {. }
$$

$U_{i}($ Zellumfang bei Grenzplasmolyse $)=129.3$.

Nimmt man ferner an, daß die Zelle bei Turgordehnung eine symmetrische Vergrößerung nach allen Richtungen des Raumes bewirke, so sollte zwischen Zellumfang und -volumen, unabhängig von der Form der Zelle, folgende Beziehung bestehen:

$$
\left(\frac{U_{1}}{U_{2}}\right)^{3}=\frac{V_{1}}{V_{2}}
$$

(XII),

worin $U_{1}$ und $U_{2}$ den Zellumfang und $V_{1}$ und $V_{2}$ das Zellvolumen bedeuten. Die nach dieser Formel und unter Anwendung der obigen Ziffer von $U_{i}$ berechneten Werte von $\phi$ sind in der vierten Spalte der Tabelle angegeben. Nimmt man für $\phi_{w}$ die Ziffer 1.34 an, so ergibt sich aus der Kurve in Fig. 5 folgendes:

$$
\begin{aligned}
& f C_{i}=3.85, \\
\therefore \quad & f=17.1 .
\end{aligned}
$$

Unter Anwendung dieser Ziffern läßt sich nach der Formel (XI) der Quotient $\phi$ für verschiedene $C_{e x}$-Werte berechnen. In Fig. 7 sind

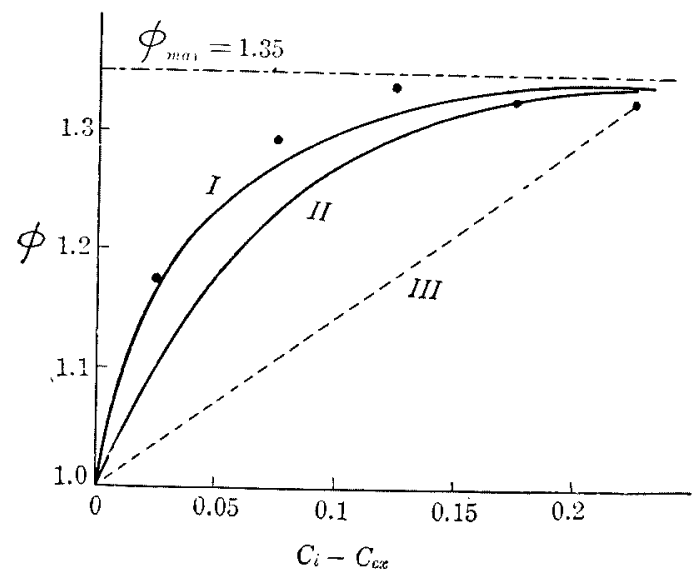

Fig. 7 die gefundenen Werte mit Punkten und der Kurve I, und die berechneten mit der Kurve II dargestellt. Wie man sieht, liegen die beiden Kurven in wesentlichen Zügen übereinander, obwohl hierbei die U̇bereinstimmung der Ziffern, vielleicht wegen der beschränkten Gültigkeit der von uns mit der Formel (XII) gemach- 
ten Umrechnung, nicht so ausgezeichnet ist wie bei dem vorangehend angeführten Beispiel. Die nach der Formel (5) gezeichnete Kurve III zeigt einen qualitativ ganz verschiedenen Verlauf, woraus man hier nochmals auf die Ungültigkeit der URsPRUNGschen Annahme schließen darf.

Jedenfalls glaube ich mit den obigen Belegen dargetan zu haben, daß unsere Formeln wohl zur Genüge genau die allgemeinen Charakteristika der Turgorerscheinung bei pflanzlichen Zellen wiedergeben. Des weiteren wollen wir sehen, zu welchen Folgerungen die weitere Entwicklung unserer Formeln führt. Aus (XI) läßt sich zunächst ableiten:

$$
\left.\begin{array}{rl}
C_{e x}=S & =C_{i}+\frac{1}{f} \ln \left(f C_{e n}-f C_{i}+1\right) \\
& =C_{i}+\frac{1}{f} \ln \left(\frac{f C_{i}}{\phi}-f C_{i}+1\right)
\end{array}\right\}
$$

Durch diese Gleichungen ist die Beziehung zwischen $C_{e x}$ (oder $S$ ) und $C_{\epsilon n}$ (oder $\phi$ ) vorgeschrieben, wobei der Festigkeitskoeffizient $f$ und der osmotische Grenzwert $C_{i}$ wichtige Faktoren darstellen. Sind also vier Werte, nämlich $C_{i}, V_{w}, V_{i}$ und $V$, bekannt, so kann man durch obige Formel die Saugkraft ausberechnen. Eine analoge Berechnung läßt sich auch nach der Annahme URSPRUNGs ausführen, was zwar das Prinzip der sogen. zweiten Methode der Saugkraftbestimmung von URSPRUNG und BLUM darstellt. Zur Anwendung kommt hierbei die Formel:

$$
S=\frac{C_{i}}{\phi}-\frac{C_{i}}{\phi_{w}\left(\phi_{w}-1\right)}(\phi-1)
$$

welche man aus der Gleichung (4) unter Berücksichtigung der Beziehung: $S=C_{e x}$ erhalten kann. Es wäre vom Interesse $\mathrm{zu}$ sehen, ob und in wieweit die nach URSPRUNG und BLUM berechneten Werte von denjenigen verschieden ausfallen, die sich aus unserer Theorie ergeben. Die bezüglich der Ergebnisse von STow bei Nitella ausgeführte Berechnung ergab die Ziffern, die in der Tabelle 3 angegeben sind. Wie ersichtlich,
Tabelle 3. Saugkraft von NitellaZelle (I. STow)

\begin{tabular}{c|c|c|c}
\hline $\begin{array}{c}\phi \\
\text { (gef.) }\end{array}$ & $\begin{array}{c}S \\
\text { (gef.) }\end{array}$ & $\begin{array}{c}S \\
\text { [ber. nach } \\
\text { (XIII)] }\end{array}$ & $\begin{array}{c}S \\
\text { [ber. nach } \\
(6)]\end{array}$ \\
\hline 1.40 & 0.00 & 0.0 & 0.0 \\
1.37 & 0.06 & 0.08 & 0.02 \\
1.37 & 0.08 & 0.08 & 0.02 \\
1.35 & 0.10 & 0.11 & 0.03 \\
1.33 & 0.12 & 0.13 & 0.05 \\
1.31 & 0.14 & 0.15 & 0.06 \\
1.29 & 0.16 & 0.16 & 0.07 \\
1.27 & 0.18 & 0.18 & 0.09 \\
1.25 & 0.20 & 0.19 & 0.10 \\
1.20 & 0.22 & 0.22 & 0.14 \\
1.14 & 0.24 & 0.24 & 0.18 \\
1.07 & 0.26 & 0.26 & 0.23 \\
1.02 & 0.28 & 0.28 & 0.27 \\
1.00 & 0.285 & 0.285 & 0.285 \\
& & &
\end{tabular}


stimmen die durch unsere Gleichung (XIII) erhaltenen Ziffern befriedigend mit den gefundenen überein, während diejenigen nach der URSPRUNGschen Formel sichtlich davon abweichen.

Ferner entnimmt man aus den Arbeiten von URSPRUNG und BLum $(1916,1920)$ folgende zwei Beispiele.

1) Blattepidermiszelle von Hedera.

Experimentell ermittelt: $V=31509, V_{i}=21799, V_{w}=34779$, $C_{i}=0.78$ (Mol. Rohrzucker).

Berechnet nach URSPRUNG und BLUM: $S=0.17$.

Berechnet nach unseren Formeln :

$$
\begin{aligned}
& \phi_{w}=1.59, \quad f C_{i}=2.45 \text {, } \\
& \phi=1.446, \quad f=3.14, \quad S=0.332 \text {. }
\end{aligned}
$$

2) Markzelle des Internodiums von Impatiens noli-tangere.

Experimentell ermittelt: $V=14122, V_{i}=13209, V_{w}=14779$, $C_{i}=0.38$ (Mol. Rohrzucker).

Berechnet nach URSPRUNG und BLUM : $S=0.152$,

Berechnet nach unseren Formeln:

$$
\begin{array}{ll}
\phi_{w}=1.12, & f C_{i}=9.4, \\
\phi=1.069, & f=24.7, \quad . S=0.342 .
\end{array}
$$

Die Diskrepanz zwischen den aus den beiden Theorien berechneten Werten ist ganz beträchtlich. Der wiederholt gemachte Hinweis auf die Ungültigkeit der URsPRUNGschen Annahme erübrigt hier die nähere Auseinandersetzung darüber, daß die dadurch ausberechneten Ziffern kaum der Wirklichkeit entsprechen können.

Kommen wir zurück zu unseren Gleichungen. Aus (I) und (IX) bezw. (I) und (XIII) folgt:

$$
\begin{aligned}
T & =C_{i}-C_{e x}-\frac{1}{f}\left[1-e^{-f\left(C_{i}-C_{e x x}\right)}\right] \\
& =C_{i}-S-\frac{1}{f}\left[1-e^{-f\left(C_{i}-S\right)}\right]
\end{aligned}
$$

und

$$
\begin{aligned}
T & =C_{e n}-C_{i}-\frac{1}{f} \ln \left[f C_{e n}-f C_{i}+1\right] \\
& =\frac{C_{i}}{\phi}-C_{i}-\frac{1}{f} \ln \left[\frac{f C_{i}}{\phi}-f C_{i}+1\right]
\end{aligned}
$$

(XV).

Die Gleichung (XIV) drückt die funktionelle Beziehung zwischen $T$ und $C_{e x}$ oder $S$, und die Gleichung (XV) diejenige zwischen $T$ und $C_{c n}$ oder $\phi$ aus. Auch hier sind der Festigkeitskoeffizient $f$ und der 
osmotische Grenzwert $C_{i}$ als wesentliche Faktoren enthalten. Die Gleichung (XV) gibt differenziert:

$$
\frac{d T}{d V}=\frac{\frac{f C_{i}{ }^{2}}{V_{i}}(\phi-1)}{\phi^{3}-f C_{i} \phi^{2}(\phi-1)},
$$

woraus man sieht, daß das Verhältnis zwischen der Zunahme des Turgordrucks und der des Zellvolumens keineswegs, wie URsPRUNG u.a. annehmen, eine Konstante, sondern eine recht komplizierte Funktion von $\phi$ ist.

Ferner ergibt sich aus (XIII) :

$$
\begin{gathered}
\frac{d \phi}{d C_{e x}}=\frac{f C_{i} \phi^{2}-\phi^{2}-f C_{i} \phi}{C_{i}} \\
\text { oder } \quad \frac{d \phi}{d\left(C_{i}-C_{e x}\right)}=\frac{\phi^{2}-f C_{i} \phi^{2}+f C_{i} \phi}{C_{i}}
\end{gathered}
$$

Bezeichnet man hierbei

$$
\frac{f C_{i}}{f C_{i}-1}=\phi_{\max }
$$

so wird die Formel (XVI) wie folgt umgeformt:

$$
\frac{d \phi}{d\left(C_{i}-C_{e x}\right)}=\phi\left(\phi_{\max }-\phi\right) \frac{f}{\phi_{\max }}
$$

Durch die Gleichungen (XVI) und (XVIII) ist das Verhältnis zwischen der Neigung der $\phi_{-}\left(C_{1}-C_{t s}\right)$-Kurve und dem Wert $\phi$ ausgedrückt. Die Bedeutung des Koeffizienten $\phi_{\max }$ wird aus folgender Überlegung einleuchtend. Da

$$
W=C_{i}-C_{e n}=C_{i}-\frac{C_{i}}{\phi}
$$

ist, so läßt sich die Formel (VII) wie folgt umschreiben:

$$
\frac{d W}{d\left(C_{i}-C_{e x}\right)}=1-f C_{i}+\frac{f C_{i}}{\phi}
$$

Ist in dieser Formel $\phi=\frac{f C_{i}}{f C_{i}-1}$, so wird $\frac{d W}{d\left(C_{i}-C_{e x}\right)}=0$, d.i. die Zelle ist nicht mehr dehnbar, oder in anderen Worten: der Grad der Turgordehnung erreicht dabei die maximale Grenze. $\phi_{\max }$ stellt somit den maximal möglichen, hypothetischen Wert dar, zu welchem der Volumquotient $\phi$ bei fortschreitender Turgordehnung asympto- 
tisch nähert.1) Die Formel (XVIII) enthält nun eine sehr bemerkenswerte Schlußfolgerung, daß nämlich die $\phi-\left(C_{i}-C_{e x}\right)$-Kurve in einem Koordinatensystem, dessen Ordinate den $\phi$-Wert und Abszisse den $\left(C_{i}-C_{e x}\right)$-Wert darstellt, eigentlich $S$-förmig, und zwar mit einem Wendepunkt an $\phi=\frac{\phi_{\max }}{2}$ geschweift sein muß. (Vergl. Fig. 8.) Inzwischen ist $\phi$ bei Grenzplasmolyse gleich 1, und, soweit die Zelle nicht plasmolysiert ist, kann $\phi$ überhaupt nicht kleiner als 1 sein. Ist also $\frac{\phi_{\max }}{2}$ nicht größer als 1 , d.i. $\phi_{\max }$ nicht größer als 2, so kann im Bereich von $\left(C_{i}-C_{e x}\right)>0$ kein Wendepunkt zur Beobachtung kommen. Aus (XVII) und ferner aus (XI') (vergl. auch die Kurvendarstellung in Fig. 5) folgt es, daßs die Bedingung $\phi_{\max }<2$ auch wie folgt ausgedrückt werden kann.

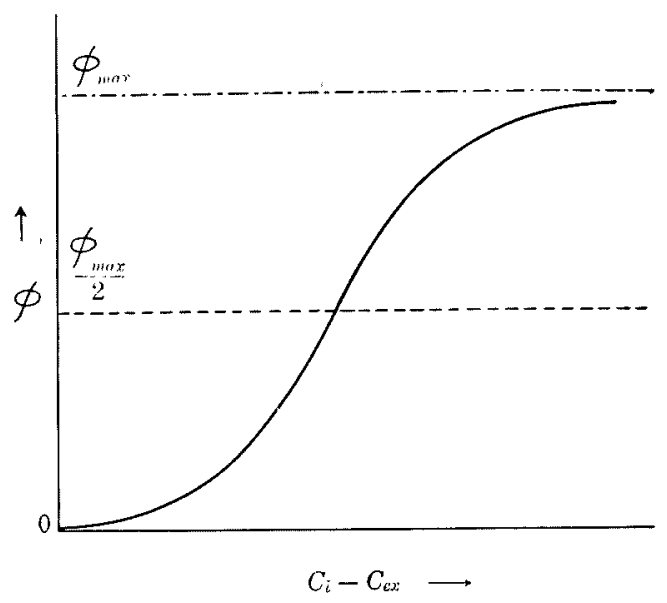

Fig. 8

$$
\begin{gathered}
f C_{i}>2 \\
\phi_{w}<1.76
\end{gathered}
$$

Daß die $\phi_{-}\left(C_{i}-C_{e x}\right)$-Kurve bei Nitelia- und Taraxacum-Zellen keinen Wendepunkt aufweist, kann dadurch erklärt werden, daß dabei der Wert $\phi_{w}$ ebenfalls kleiner als 1.76 ist.

Um kennen zu lernen, ob und in welcher Grenze der Wert $\phi_{w}$ sowie der Festigkeitskoeffizient $f$ bei verschiedenen Zellen variiert, sei hier der Befund von H. R. OPPENHEIMER (1930) angeführt, der die Membrandehnbarkeit von verschiedenen Zellen durch Messung des Zellumfangs verglichen hat. Der von diesem Autor als Maßstab der Dehnbarkeit angewandte Quotient:

Zellumfang bei wassergesättigtem Zustand-Zellumfang bei Grenzplasmolyse

$$
\text { Zellumfang bei Grenzplasmolyse }
$$

1) Wenn in der Formel $\left(\mathrm{XI}^{\prime}\right) f C_{i}$ sehr groB ist, d.i. $-\frac{V_{i}}{V_{w}} \doteqdot 1$, so wird $\phi_{w} \doteqdot \phi_{\max }$, sonst muB $\phi_{w}$ stets kleiner als $\phi_{\max }$ sein.

Bezeichnet man den Wasserwert bei $C_{e x}=0$ mit $W_{w}$, so kann man denselben auch dann gleich $W_{\max }$ setzen, wenn $f C_{i}$ sehr groß ist. Denn nach (VI) bei $\alpha=1$ ist $W_{\max }=\frac{1}{f}$, und nach (VIII) bei $C_{e x}=0$ ist $W_{w}=\frac{1}{f}-\frac{1}{f} e^{-f C_{i}}$. 
variierte bei verschiedenen Objekten zwischen null (Blattepidermen von Hedera, Musa, Lamium u.a.) und 0.22, welch letzterer Wert bei Blattepidermen von Bellis perennis gefunden wurde. Nimmt man hierbei vereinfachenderweise an, daß die Dehnung der Zellwand nach allen Richtungen des Raumes gleichmäßig erfolge, so sollten die minimale und die maximale Grenze von $\phi_{w} 0$ bzw. 1.816 (also größer als 1.76) sein. Hieraus berechnen sich der maximale und minimale Wert von $f C_{i}$ zu $\infty$ bzw. 1.9. Da der höchste osmotische Wert der Pflanzenzellen etwa $1.4 \sim 1.9 \mathrm{Mol}$. Rohrzucker (=60 100 Atm.) entspricht,") so könnte man als den denkbar maximalen und den minimalen Wert von $f$ die Ziffer $\infty$ bzw. 1.4 1 annehmen. In der Tabelle 4 sind die Werte, $\phi_{w}, C_{i}$ und $f$ bei den schon zitierten Beispielen zusammengestellt, woraus man sieht, daß der Festigkeitskoeffizient, im Gegensatz zu anderen Größen, je nach den Arten der Zellen ganz verschieden ausfällt.

Tabelle 4

\begin{tabular}{l|c|c|c}
\hline & $\varphi_{w}$ & $C_{i}$ & $f$ \\
\hline Nitella & 1.40 & 0.285 & 11.9 \\
Taraxacum & 1.34 & 0.225 & 17.1 \\
Hedera & 1.59 & 0.78 & 3.14 \\
Impatiens & 1.12 & 0.38 & 24.7
\end{tabular}

Aus den schon gesagten ersieht man, daß bei äußerst dehnbaren Zellen, bei denen $\phi_{w}=1.816$ sein könnte, der Wendepunkt der $\phi_{-}\left(C_{i}-\right.$ $\left.C_{e x}\right)$-Kurve wohl in der Nähe der Grenzplasmolyse zu beobachten ist. Dies kann aber gewöhnlich kaum der Fall sein, weil $\phi_{w}$ bei meisten Zellen kleiner als 1.76 ist. Es sei hier darauf aufmerksam gemacht, daß wir in der bisherigen Darlegung nur die Erscheinungen bei nicht plasmolysiertem Zustand betrachtet haben. Bei plasmolysierten Zellen, bei denen die Zellwand gar keinen Einfluß mehr auf die Volumenänderung des Zellsaftes ausübt, wird natürlich der Festigkeitskoeffizient der Zellmembran ganz belanglos. Aus der Arbeit von K. HöFler (1917b) geht hervor, daß für plasmolysierte Zellen im großen und ganzen folgende Proportionalität besteht.

$$
\frac{C_{e x_{1}}}{C_{e x_{2}}}=\frac{C_{e n_{1}}}{C_{e n_{2}}}=\frac{V_{2}}{V_{1}}
$$

Dies besagt, daß bei plasmolysierten Zellen die Volumenänderung praktisch ohne Widerstand vor sich geht. Wenn man für solche Fälle $f=0 \quad$ (Festigkeitskoeffizient der Plasmaschicht) setzen darf, so ergibt sich aus (XVI) die Gleichung:

$$
\frac{d \phi}{d\left(C_{i}-C_{e x}\right)}=\frac{\phi^{2}}{C_{i}}
$$

1) Vergl. z.B. O. RENNER (1915) u. H. WALter (1931). 
Nach dieser Formel soll die $\phi-\left(C_{i}-C_{e x}\right)$-Kurve bei plasmolysierten Zellen nicht $S$-förmig gestaltet, sondern, etwa wie die Kurve in Fig. 2, einfach nach oben gekrümmt sein. Eine solche Kurve finden wir wirklich bei plasmolysierter Zelle von Spirogyra (H. WALTER 1923) und Nitella (I. STow. 1936), welche letztere uns besonders deutlich zeigt, daß die Dehnungskurve an beiden Seiten der Grenzplasmolyse gründlich verschiedenen Verlauf aufweist.

Die Formeln (X), (XIII) und (XV) drücken die Größen $C_{c n}$, $S$ und $T$ ebenfalls als Funktion von $\phi$ aus. Sind bei einer Zelle der osmotische Grenzwert $C_{i}$ und der Quotient $\phi_{w}$ und folglich der Festigkeitskoeffizient $f$ bekannt, so kann man durch diese Formeln für verschiedene Werte von $\phi$ die entsprechende Größe von $C_{e n}, S$ und $T$ ausrechnen. Die Beziehungen zwischen diesen 4 osmotischen Größen läßst sich nun ganz übersichtlich nach dem Vorgehen von HöFLER (1920) graphisch darstellen, indem man $C_{c n}, T$ und $S$ gleichzeitig auf die Ordinate und $\phi$ auf die Abszisse eines Koordinatensystems aufträgt. Fig. 9 stellt ein solches Diagramm für die von STow

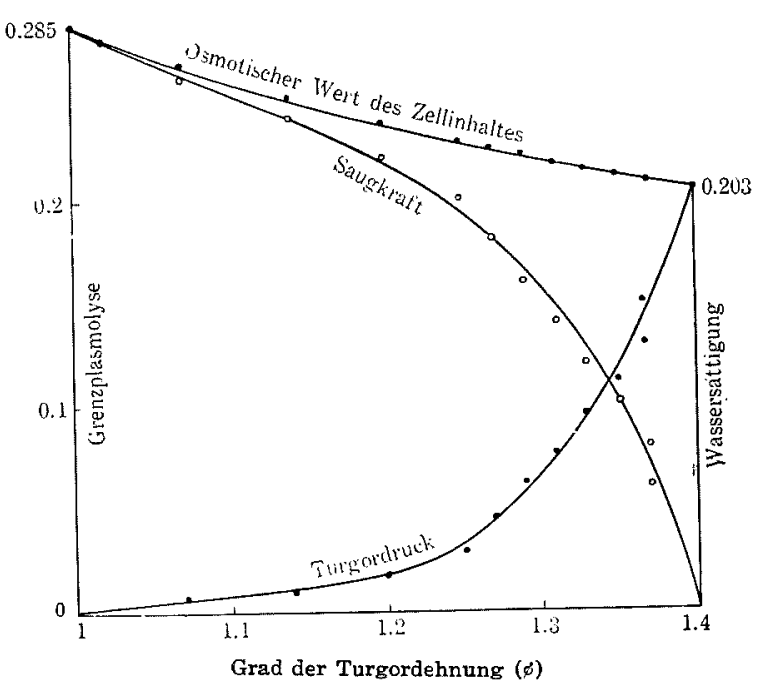

Fig. 9 untersuchte NitellaZelle dar, woraus wir sehen, daß die Saugkraft- sowie die Turgordruck-Kurve ebenfalls exponentiellen Verlauf aufweist. Daß die Saugkraftund die TurgordruckKurve im HöFLERDiagramm nicht gerade, wie früher HÖFLER in Anlehnung an die Annahme URSPRUNGs geltend machte, sondern prinzipiell ex-

ponentielle Charaktere besitzen sollten, hat neulich A. FREY-WYssLING (1935) auf Grund der von OPPENHEIMER ermittelten Dehnungskurve gemutmaßt. Die mathematischen Charaktere der Kurven, worüber FREY-WYSSLING seinerzeit nichts bestimmtes aussagen konnte, sind wohl erst durch unsere Gleichungen konkret zum Ausdruck gebracht.

Schließlich muß aber betont werden, daß die Gültigkeit unserer Theorie selbstverständlich durch mehrere Postulate bedingt ist, die wir schon an verschiedenen Stellen in unserer Auseinandersetzung 
vorgeschrieben haben. Es wäre zweckmässig, hier nochmals auf unsere Voraussetzungen hinzuweisen:

1) Es wird nur isolierte, in keinem Außsendruck oder -Zug unterworfene Zelle betrachtet.

2) Die Zelle soll eine genügend große Zentralvakuole und genügend dünne und gegen Osmotika vollkommen semipermeable protoplasmatische Schicht besitzen, sodaß bezüglich des osmotischen Wertes des Zellsaftes und des Zellvolumens das BoYLE-MARIOTTEsche Proportionalitätsgesetz gelten kann.

3) Weder Wachstum noch die Veränderung der Menge dor osmotisch aktiven Zellsubstanzen soll stattfinden.

4) Die ganze Zellmembran, mit dem anliegenden Plasmabelag, muß ein einheitliches System darstellen, und weder die Dehnbarkeit der Zellwand noch der osmotische Wert des Zellsaftes soll keine polare Differenzierung aufweisen.

5) Es soll sich im Laufe der Turgordehnung keine plötzliche qualitative Veränderung der Membraneigenschaft, wie Zerreißen, übermäßige plastische Dehnung oder Plasmolyse ereignen.

6) Bei turgeszenzlosem Zustand sei die Zellwand widerstandslos dehnbar; d.i. bei Grenzplasmolyse sei $\frac{d W}{d\left(C_{i}-C_{e x}\right)}=1$ oder $\alpha=1$, (vergl. Gleichungen (V') und (VI)).

Einer besonderen Beachtung verdient hierbei die zuletzt erwähnte Voraussetzung über die Dehnbarkeit bei turgeszenzlosem Zustand. Während bei sehr dehnbaren parenchymatischen Zellen, wie wir es bei Nitella- und Taraxacum-Zelle gesehen haben, die Annahme, a sei 1 , sich mit genügender Annäherung bewährt, so kann sie doch augenscheinlich nicht auf die Fälle der viel weniger dehnbaren sklerenchymatischen Zellen verallgemeinert werden. Ist $a<1$, d.i., behält die Zellwand auch bei Grenzplasmolyse noch eine gewisse Sprödität bei, so hat man zur Charakterisierung der Abhängigkeit der Turgordehnung von der Außenkonzentration nicht nur den Festigkeitskoeffizienten $f$ allein, sondern auch den Wert $a$ in Berücksichtigung zu ziehen. Aus unserer Grundformel (V) lassen sich sodann die Gleichungen ableiten, die viel allgemeiner als die bisher angeführten gültig sein können. Übersichtlichkeitshalber seien unten solche Gleichungen zusammenfassend angegeben.

1) Der Wasserwert:

$$
W=\frac{a}{f}\left[1-e^{-f\left(C_{i}-C_{e x}\right)}\right]=\frac{\alpha}{f}\left[1-e^{-f\left(C_{i}-S\right)}\right] \quad(\mathrm{XIX})
$$


2) Der osmotische Wert des Zellinhaltes:

$$
\begin{array}{ll}
C_{e n}=C_{i}-\frac{a}{f}\left[1-e^{-f\left(C_{i}-C_{e x}\right)}\right]=C_{i}-\frac{a}{f}\left[1-e^{-f\left(C_{i}-S\right)}\right](\mathrm{XX}), \\
C_{w}=C_{i}-\frac{\alpha}{f}\left[1-e^{-f C_{i}}\right] & \left(\mathrm{XX}^{\prime}\right) .
\end{array}
$$

3) Die Saugkraft:

$$
\begin{aligned}
S=C_{e x} & =\frac{1}{f} \ln \left[\frac{f C_{i}}{\alpha \phi}-\frac{f C_{i}}{\alpha}+1\right]+C_{i} \\
& =\frac{1}{f} \ln \left[\frac{f}{\alpha} C_{e n}-\frac{f C_{i}}{\alpha}+1\right]+C_{i}
\end{aligned}
$$

4) Der Turgordruck:

$$
\begin{aligned}
T & =C_{i}-S-\frac{\alpha}{f}\left[1-e^{-f\left(C_{i}-S\right)}\right] \\
& =C_{i}-C_{e x}-\frac{\alpha}{f}\left[1-e^{-f\left(C_{i}-C_{e x}\right)}\right] \\
T & =C_{e n}-C_{i}-\frac{1}{f} \ln \left[\frac{f}{\alpha} C_{e n}-\frac{f}{\alpha} C_{i}+1\right] \\
& =\frac{C_{i}}{\phi}-C_{i}-\frac{1}{f} \ln \left[\frac{f C_{i}}{\alpha \phi}-\frac{f C_{i}}{\alpha}+1\right]
\end{aligned}
$$

5) Der Grad der Turgordehnung:

$$
\begin{aligned}
& \phi=\frac{f C_{i}}{f C_{i}-\alpha+\alpha e^{-f\left(C_{i}-C_{e x}\right)}} \\
& \phi_{w}=\frac{f C_{i}}{f C_{i}-\alpha+\alpha e^{-f C_{i}}} \\
& \phi_{\max }=\frac{f C_{i}}{f C_{i}-\alpha} \\
& \frac{d \phi}{d\left(C_{i}-C_{e x}\right)}=\frac{f C_{i} \phi-f C_{i} \phi^{2}+\alpha \phi^{2}}{C_{i}}=\phi\left(\phi_{\max }-\phi\right) \frac{f}{\phi_{\max }} \text { (XXVI). }
\end{aligned}
$$

Die früher angegebenen Formeln stellen nur die Spezialfälle der obigen Gleichungen dar, und zwar mit der Beschränkung: $a=1$. Aus (XXVI) folgt für den grenzplasmolytischen Zustand, bei welchem also $\phi=1$ ist, die Beziehung:

$$
\left[\frac{d \phi}{d\left(C_{i}-C_{e x}\right)}\right]_{i}=\frac{a}{C_{i}}
$$




$$
\therefore \quad \alpha=C_{i}\left[\frac{d \phi}{d\left(C_{i}-C_{e x}\right)}\right]_{i}
$$

Es stellt sich heraus, daß man den Koeffizienten $\alpha$ aus $C_{i}$ und der Tangente der $\phi_{-}\left(C_{i}-C_{e^{x}}\right)$-Kurve in der Nähe der Grenzplasmolyse ermitteln kann. Sind $\alpha, \phi_{w}$ und $C_{i}$ bekannt, so läßt sich aus (XXIV') auch der Wert $f$ feststellen. Die Berechnung der einzelnen osmotischen Größen aus den anderen Werten kann sodann auf ganz analoge Weise geschehen, wie es oben an einfacheren Beispielen gezeigt wurde. Leider sind wir zur Zeit nicht im Stande, die obigen Ausführungen mit experimentellen Daten zu belegen, weil über die quantitativen Verhältnisse, die bei der Turgordehnung der spröden Zellen obwalten, noch keine genauen Angaben vorliegen.

Es liegt ja auf der Hand, daß allgemein die Turgorerscheinung der Zellen wegen manchen Umständen noch viel komplizierter sein kann, als es hier angenommen wurde. Bei Behandlung solcher Fälle hat man natürlich jeweils den spezifischen Eigenschaften der Zellen und besonderen Umständen Rechnung zu tragen, wobei unsere Theorie als solche nicht unmittelbar geltend gemacht werden kann. So beschränkt die Tragweite unserer Theorie für die Kenntnis der allgemeinen osmotischen Erscheinungen sein mag, so möchte ich doch zu behaupten wagen, daß die unter einfacheren Verhältnissen gewonnenen Erfahrungen als ein wichtiger Anhalt und Wegweiser zum weiteren Vordringen dienen könnten.

Meinem hochverehrten Lehrer, Herrn Prof. K. Shibata, bin ich für die freundliche Unterstützung bei dieser Arbeit zu aufrichtigem Dank verpflichtet. Herzlichsten Dank schuldige ich ferner Herrn Prof. T. SAKA Mura im botanischen Institut der Kaiserl. Universität zu Sapporo für das fördernde Interesse und wertvolle Kritik, die er mir während dieser Arbeit vielfach durch briefliche Korrespondenz entgegenbrachte.

\section{Übersicht der gebrauchten Bezeichnungen}

$C_{e n}: \quad$ Osmotischer Wert des Zellinhaltes.

$C_{e x}$ : Osmotischer Wert der mit der Zelle im Gleichgewicht stehenden AuBenlösung.

$C_{i}$ : Osmotischer Wert des Zellinhaltes bei Grenzplasmolyse=Osmotis:her Wert der Außenlösung bei Grenzplasmolyse.

$C_{w}$ : Osmotischer Wert des Zellinhaltes in wassergesättigtem Zustand.

$V: \quad$ Volumen der Zelle, deren osmotischer Wert $C_{e n}$ ist.

$V_{i}$ : $\quad$ Volumen der Zelle bei Grenzplasmolyse.

$V_{w}$ : Volumen der Zelle in wassergesättigtem Zustand.

$T: \quad$ Turgordruck der Zelle, deren osmotischer Wert $C_{e n}$ ist.

$S$ : $\quad$ Saugkraft der Zelle, deren osmotischer Wert $C_{e n}$ ist. 
$W: \quad$ „Wasserwert" der Zelle, deren osmotischer Wert $C_{e n}$ ist. Wasserwert bedeutet denjenigen Bruchteil des osmotischen Wertes, der bei Überfỉhrung der Zelle von grenzplasmolytischer Lösung $\mathrm{zu}$ einer Lösung mit dem osmotischen Wert von $C_{e x}$ zur Wasseraufnahme aufgewandt wird.

$\phi: \quad$ Grad der Turgordehnung: d.i. $=\frac{V}{V_{i}}$.

$\phi_{w}: \quad$ Grad der Turgordehnung in wassergesättigtem Zustand : $\frac{V_{w}}{V_{i}}$.

$\phi_{\text {ma: }}$ : Maximaler Grenzwert von $\phi$.

$\alpha$ : $\quad$ Grenzwert von $\frac{d W}{d\left(C_{i}-C_{e x}\right)}$ bei der Grenzplasmolyse. Bei parenchymatischen Zellen darf man $\alpha=1$ setzen.

$f: \quad$,Festigkeitskoeffizient" Beziehung steht.

$$
\frac{d W}{d\left(C_{i}-C_{e x x}\right)}=\alpha-f W
$$

\section{Literatur}

Buch, L. 1935. Beih. z. Bot. Zentralbl., 53, A, 340,

Frey-Wyssling, A. 1935. Die Stoffausscheidung der höheren Pflanzen. Berlin.

Gamma, H. 1932. Protopl., 16, 489.

Heyn, A. N. J. 1933. Protopl., 19, 78.

Hofe, F. V. 1933. Planta, 20, 354.

Hoffmann, C. 1932. Planta, 16, 413.

- 1935. Protopl., 24, 286.

Hëfler, K. 1917 a. Ber. d. deut. bot. Ges., 35, 723.

- 1917 b. Anz. Kaiserl. Akad. d. Wiss. Math. -Nat., 54, 225.

- 1920. Ber. d. deut. bot. Ges., 38, 288.

Lepeschkin, W. W. 1907. Beih. z. Bot. Zentralbl., I. Abt., 21, 60.

Oppenheimer, H. R. 1930 . Ber. d. deut. bot, Ges., 48, 192, (150).

Pringsheim, E. G. 1931. Jahrb. f. wiss. Bot., 74, 749.

Renner, O. 1915. Jahrb. f. wiss. Bot., 56, 617.

Sakamura, T. 1937. Cytologia, Fujii Jubil. Vol., 115.

Stow, I. 1936. Bot. and Zool., 4, 1330. (Japanisch).

Ursprung, A, 1926. Planta, 2, 640.

- 1932. H indwörterb. d. Naturwiss., 2. Aufl., Bd. 7.

- u. G. Blum. 1916. Ber. d. deut. bot. Ges., 34, 525.

- u. G. Blum. 1920. Biol. Zentralbl., 40, 193.

Walter, H. 1923. Jahrb. f. wiss. Bot., 62. 145.

- 1931. Die Hydratur der Pflanze. Jena. 